\title{
THE OHSAWA-TAKEGOSHI EXTENSION THEOREM ON SOME UNBOUNDED SETS
}

\author{
ŻYWOMIR DINEW
}

\begin{abstract}
We use a method of Berndtsson to obtain a simplification of Ohsawa's result concerning extension of $L^{2}$-holomorphic functions. We also study versions of the Ohsawa-Takegoshi theorem for some unbounded pseudoconvex domains, with an application to the theory of Bergman spaces. Using these methods we improve some constants, that arise in related inequalities.
\end{abstract}

\section{$\S 0$. Introduction}

In [O-T] Ohsawa and Takegoshi obtained the following result:

THEOREM 1. Let $\nu$ be a plurisubharmonic function on a bounded pseudoconvex domain $D \subset \mathbb{C}^{n}$, let $H \subset \mathbb{C}^{n}$ be a complex hyperplane and let $f$ be a holomorphic function on $D \cap H$ satisfying

$$
\int_{D \cap H}|f(z)|^{2} e^{-\nu(z) \mid D \cap H} d \lambda^{2 n-2}<\infty
$$

(Here $\lambda^{2 n-2}$ stands for the $2 n-2$-dimensional Lebesgue measure). Then there exists a holomorphic function $F$ on $D$, such that $F_{\mid D \cap H}=f$ and

$$
\int_{D}|F(z)|^{2} e^{-\nu(z)} d \lambda^{2 n} \leq C_{0} \int_{D \cap H}|f(z)|^{2} e^{-\nu(z)_{\mid D \cap H}} d \lambda^{2 n-2} .
$$

Here $C_{0}$ is a constant, that depends only on the diameter of $D$.

The proof used $\bar{\partial}$ methods and somehow complicated theory of Kähler metrics. Because of the great importance of this result and the remarkable applications (for example it yields interesting estimates of the Bergman kernel), it motivated plenty of generalizations and new proofs. Most of the research is carried in the direction of changing $D \cap H$ to varieties, or stating

Received March 14, 2006.

Revised December 15, 2006.

2000 Mathematics Subject Classification: 32D15, 32A36. 
the theorem in the setting of bundles. It seems that the case, when one keeps $H$ to be a hyperplane, but relaxes the condition of boundedness of $D$, is far less studied, although in many papers Ohsawa-Takegoshi type theorems are proved for unbounded $D$ 's, with various restrictions on the domain (see e.g., $[\mathrm{Be} 2],[\mathrm{D}-\mathrm{H}])$. Since it is clear that one cannot expect an Ohsawa-Takegoshi type estimate for any unbounded pseudoconvex $D$, it seems interesting to characterize these, on which some version of the theorem holds. One possible goal of such characterization is explained below.

Of course, when we deal with unbounded domains, $C_{0}$ cannot depend on the diameter of the domain, so we must look for another quantitative characteristic of the set.

In [O1] the following generalization of Theorem 1 was established.

Theorem 2. Let $D, \nu$ and $H$ be as in Theorem 1 and let $d(z, H)$ be the Euclidean distance from $z \in \mathbb{C}^{n}$ to the hyperplane $H$. Then, for any plurisubharmonic function $\varphi$ on $D$, such that $\varphi(z)+2 \log d(z, H)$ is bounded from above, there exists a constant $C_{1}$, depending only on $\sup _{D}(\varphi+$ $2 \log d(\cdot, H))$, such that for any holomorphic function $f$ on $D \cap H$, satisfying

$$
\left.\int_{D \cap H}|f(z)|^{2} e^{-\nu(z)_{\mid D \cap H}-\varphi(z)}\right|_{\mid D \cap H} d \lambda^{2 n-2}<\infty,
$$

there exists a holomorphic function $F$ on $D$, satisfying $F_{\mid D \cap H}=f$ and

$$
\int_{D}|F(z)|^{2} e^{-\nu(z)} d \lambda^{2 n} \leq C_{1} \int_{D \cap H}|f(z)|^{2} e^{-\nu(z)_{\mid D \cap H}-\varphi(z)_{\mid D \cap H}} d \lambda^{2 n-2} .
$$

Although stated for bounded domains, Theorem 2 holds also for some general ones (the approximation argument, which is by no means new, is given in Section 1).

About a year later Berndtsson simplified the proof of Theorem 1. The main advantage of his method is that it avoids the Kähler metrics, as well as the construction of local extensions.

The main goal of this paper is to show, that Berndtsson's arguments, with minor modifications, can be used to prove Theorem 2 with the constant $C_{1}=4 \pi e^{\sup _{D}(\varphi+2 \log d(\cdot, H))}$, much better than in the original paper of Ohsawa. An alternative proof of this theorem was given in $[\mathrm{D}-\mathrm{H}]$. The author believes that the arguments contained in this paper are simpler.

Let $G_{\Omega}(z, \cdot)$ be the negative Green function of a domain $\Omega \subset \mathbb{C}$, with pole at $z$. 
Denote by

$$
c(\Omega, z)=\exp \left(\lim _{\zeta \rightarrow z} G_{\Omega}(z, \zeta)-\log |z-\zeta|\right)
$$

the so called logarithmic capacity.

One defines the Bergman kernel as $K_{\Omega}(z)=\sup \frac{|f(z)|^{2}}{\int_{\Omega}|f(z)|^{2} d \lambda^{2}}, f \in \mathcal{O}(\Omega)$, $f$ is not identically 0 .

The following version of Theorem 2 seems to be more handy

Theorem 3. Let $D \subset \Omega \times \mathbb{C}^{n-1}$, where $\Omega \subset \mathbb{C}$ is a planar domain. Let $0 \in D$. Let $\nu, f, F$ be as above. Then

$$
\begin{aligned}
& \int_{D}|F(z)|^{2} e^{-\nu(z)} d \lambda^{2 n} \\
& \quad \leq \frac{4 \pi}{(c(\Omega, 0))^{2}} \int_{D \cap\left\{z_{1}=0\right\}}\left|f\left(z^{\prime}\right)\right|^{2} e^{-\nu\left(z^{\prime}\right) \mid D \cap\left\{z_{1}=0\right\}} d \lambda^{2 n-2}\left(z^{\prime}\right) .
\end{aligned}
$$

One just needs to substitute $2\left(G_{\Omega}\left(0, z_{1}\right)-\log \left|z_{1}\right|\right)$ for $\varphi\left(z_{1}, z^{\prime}\right)$, which is a harmonic function in the first variable and hence pluriharmonic, to see that Theorem 3 follows directly from Theorem 2. This estimate is in some sense optimal in terms of $\Omega$, because the capacity is nonzero exactly when $\Omega$ has nonpolar complement in $\mathbb{C}$, which in the one-dimensional situation is equivalent to the existence of nonzero square-integrable holomorphic function.

As noted by many, the Ohsawa-Takegoshi estimate is some kind of multidimensional estimate for the Bergman kernel. In dimension one we have the following problem (still open).

ConjeCture. (Suita) For $\Omega \subset \mathbb{C}$-open and bounded (see $[\mathrm{S}]$, where the problem is investigated in the setting of Riemann surfaces) $\pi K_{\Omega}(z) \geq$ $(c(\Omega, z))^{2}$.

Applying Theorem 3 to the one dimensional case, with $\nu=0$ (then clearly the section $\left\{z_{1}=0\right\} \cap D$ degenerates to a single point and instead of the integral we have valuation on the right hand side), we get

$$
4 \pi K_{\Omega}(0) \geq(c(\Omega, 0))^{2} .
$$

To author's knowledge the best constant so far $(2 \pi)$ was obtained by Z. Błocki (see $[\mathrm{Bl}]$ ), by using slightly different methods. 
Instead of taking valuation, we could of course proceed as follows. We choose $D$ to be a Cartesian product $\Omega \times H$ ( $H$-is any bounded pseudoconvex domain, $\Omega$-as above open and one-dimensional). In this case the minimal (by norm) extension is of the form $h\left(z_{1}\right) f\left(z^{\prime}\right)$, where $f$ is the function to be extended (which is independent of $z_{1}$ ), $h$-is the function which realizes the supremum in the definition of the Bergman kernel and $h(0)=1$, which is an easy consequence of Fubini's theorem. We also immediately get the above estimate.

It is worth mentioning, that if we apply Berndtsson's argument (see [Be1], [Be2]) directly, by passing to the infimum, we get the constant to be $\frac{4 \pi}{\left(c_{\beta}(\Omega, 0)\right)^{2}}$, where $c_{\beta}(\Omega, 0)$ is the analytic capacity.

In $[\mathrm{W}]$ Wiegerinck showed that it is possible to construct domains $\Omega$ in $\mathbb{C}^{2}$, such that $\operatorname{dim} L^{2} \cap \mathcal{O}(\Omega)$ is any nonzero integer. The domains constructed are not pseudoconvex and any pseudoconvex domain that contains such a domain has trivial $L^{2} \cap \mathcal{O}$ space. In $[\mathrm{J}-\mathrm{P}]$ the following problem is set: does there exist a pseudoconvex domain whose $L^{2} \cap \mathcal{O}$ space is finite dimensional and non-trivial. From the full characterization of plane domains (this can be found in $[\mathrm{W}]$ ) and Theorem 3 we easily deduce the following.

Corollary. If $\Omega$ is a pseudoconvex domain in $\mathbb{C}^{2}$, for which $\exists X \in \mathbb{C}^{2}$, such that the projection $\boldsymbol{p r}_{\{\lambda X, \lambda \in \mathbb{C}\}} \Omega$ has nonpolar complement in $\mathbb{C}$, then $\operatorname{dim} L^{2} \cap \mathcal{O}(\Omega)=0$ or $\infty$. Moreover if there exists a section orthogonal to $\{\lambda X, \lambda \in \mathbb{C}\}$ with nonpolar complement, then $\operatorname{dim} L^{2} \cap \mathcal{O}(\Omega)=\infty$ (and hence almost all such sections have nonpolar complements).

This of course falls short of full characterization, since for example the following pseudoconvex Reinhardt domain

$$
\left\{(z, w) \in \mathbb{C}^{2}:|w| \leq e^{f(\ln |z|)}\right\}
$$

where

$$
f(x)= \begin{cases}\sqrt{-2 x}, & x<0 \\ -\frac{x^{2}}{2}, & x \geq 0\end{cases}
$$

has non-trivial Bergman space, but does not satisfy the conditions of the corollary. The Corollary is particularily useful for Hartogs domains, since all we have to check is that the base has nonpolar complement.

I would like to express my grattitude to Professor Zbigniew Błocki for helpful discussions and encouragement. 


\section{$\S 1$. Proof of Theorem 2}

From now on we will assume, without loss of generality, that $D \cap H$ is identical with $\left\{z_{1}=0\right\} \cap D$. Then $\log d(z, H)$ reduces to $\log \left|z_{1}\right|$. In the whole paper $\bar{\partial}_{\psi}^{*}$ will stand for the formal adjoint operator to $\bar{\partial}$, that is

$$
\langle\bar{\partial} \alpha, \beta\rangle_{L_{(0,1)}^{2}(D, \psi)}=\left\langle\alpha, \bar{\partial}_{\psi}^{*} \beta\right\rangle_{L_{(0,0)}^{2}(D, \psi)}
$$

in the notation of the Hilbert space of $L^{2}$-functions (forms) with weight $e^{-\psi}$. Then $\bar{\partial}_{\psi}^{*}$ is easily explicitly calculated as

$$
\bar{\partial}_{\psi}^{*} \alpha=-e^{\psi} \sum_{j=1}^{n} \frac{\partial}{\partial z_{j}}\left(e^{-\psi} \alpha_{j}\right) .
$$

We do almost the same as in [Be1]. The following can be found there.

Let $D$ be a smoothly bounded domain defined as the sublevel $\rho<0$ of a $\mathcal{C}^{\infty}$ and strictly plurisubharmonic function $\rho$. Let the weight function $\psi$ be plurisubharmonic and (temporarily) we will assume that it is smooth. We also temporarily assume that $\varphi$ is smooth and defined on some neighbourhood of $D$.

Then:

(†) If $g$ is a $\bar{\partial}$-closed $(0,1)$-current, defined in a neighbourhood of $\bar{D}$ and $u$ is a $L^{1}$ function in $D$, such that

$$
\int_{D} g \cdot \bar{\alpha} e^{-\psi} d \lambda^{2 n}=\int_{D} u \overline{\bar{\partial}_{\psi}^{*} \alpha} e^{-\psi} d \lambda^{2 n}
$$

for all smooth, $\bar{\partial}$-closed $(0,1)$-forms $\alpha$ on $\bar{D}$, satisfying the $\bar{\partial}$-Neumann boundary conditions, then $\bar{\partial} u=g$ in the sense of distributions.

( $\dagger \dagger$ ) If $g$ and $D$ are as above and the inequality

$$
\left|\int_{D} g \cdot \bar{\alpha} e^{-\psi} d \lambda^{2 n}\right|^{2} \leq C \int_{D}\left|\bar{\partial}_{\psi}^{*} \alpha\right|^{2} \frac{e^{-\psi}}{\tau} d \lambda^{2 n}
$$

where $\frac{1}{\tau}$ is an integrable, non-negative function, holds for all forms $\alpha$ of the above type, then there exists an $u$, such that $\bar{\partial} u=g$ (also in the sense of distributions) and

$$
\int_{D}|u|^{2} \tau e^{-\psi} \leq C
$$


$(\dagger \dagger \dagger)$ If $D, \psi$ and $\rho$ are as above, $\alpha$-fixed smooth $(0,1)$-form, satisfying the $\bar{\partial}$-Neumann boundary conditions and $w$ be a function that is smooth on some neighborhood of $D$ (or can be approximated by such functions, with control of the derivatives), then

$$
\begin{gathered}
\int_{D} w \sum_{j, k=1}^{n} \psi_{j \bar{k}} \alpha_{j} \bar{\alpha}_{k} e^{-\psi} d \lambda^{2 n}-\int_{D} \sum_{j, k=1}^{n} w_{j \bar{k}} \alpha_{j} \bar{\alpha}_{k} e^{-\psi} d \lambda^{2 n} \\
\quad+\int_{D} w\left|\bar{\partial}_{\psi}^{*} \alpha\right|^{2} e^{-\psi} d \lambda^{2 n}+\int_{D} w \sum_{j, k=1}^{n}\left|\frac{\partial \alpha_{j}}{\partial \bar{z}_{k}}\right|^{2} e^{-\psi} d \lambda^{2 n} \\
\quad+\int_{\partial D} w \sum_{j, k=1}^{n} \rho_{j \bar{k}} \alpha_{j} \bar{\alpha}_{k} e^{-\psi} \frac{d \sigma^{2 n-1}}{|\partial \rho|} \\
=2 \operatorname{Re} \int_{D} w \bar{\partial}_{\psi}^{*} \alpha \cdot \bar{\alpha} e^{-\psi} d \lambda^{2 n}+\int_{D} w|\bar{\partial} \alpha|^{2} e^{-\psi} d \lambda^{2 n} .
\end{gathered}
$$

Here $\beta \cdot \gamma$ stands for the dot product in $\mathbb{C}^{n}$ i.e., $\beta \cdot \gamma=\beta_{1} \gamma_{1}+\beta_{2} \gamma_{2}+\cdots+$ $\beta_{n} \gamma_{n}$ and $\bar{\partial}$-Neumann boundary conditions for $\alpha$ means, that $\left.\alpha\right\lrcorner \partial \rho_{\mid \partial D}=0$, or equivalently $\sum_{j=1}^{n} \alpha_{j} \frac{\partial}{\partial z_{j}} \rho=0$ on the boundary of $D$, where $\alpha=\alpha_{1} d \bar{z}_{1}+$ $\cdots+\alpha_{n} d \bar{z}_{n}$.

Note that the right hand side of the equality ( $\dagger \dagger \dagger$ ) can be written as

$$
\begin{aligned}
2 \operatorname{Re}\left\langle w \partial \bar{\partial}_{\psi}^{*} \alpha, \alpha\right\rangle_{L_{(0,1)}^{2}(D, \psi)}+\|\sqrt{w} \bar{\partial} \alpha\|_{L_{(0,2)}^{2}(D, \psi)}^{2} \\
=2\left\langle w \bar{\partial}_{\psi}^{*} \alpha, \bar{\partial}_{\psi}^{*} \alpha\right\rangle_{L_{(0,0)}^{2}(D, \psi)}-2 \operatorname{Re}\left\langle\bar{\partial}_{\psi}^{*} \alpha \bar{\partial} w, \alpha\right\rangle_{L_{(0,1)}^{2}(D, \psi)} \\
\quad+\|\sqrt{w} \bar{\partial} \alpha\|_{L_{(0,2)}^{2}(D, \psi)}^{2} \\
=2\left\|\sqrt{w} \bar{\partial}_{\psi}^{*} \alpha\right\|_{L_{(0,0)}^{2}(D, \psi)}^{2}+\|\sqrt{w} \bar{\partial} \alpha\|_{L_{(0,2)}^{2}(D, \psi)}^{2} \\
\quad-2 \operatorname{Re} \int_{D} \bar{\partial}_{\psi}^{*} \alpha(\bar{\partial} w \cdot \bar{\alpha}) e^{-\psi} d \lambda^{2 n}
\end{aligned}
$$

hence for closed $\alpha$, nonnegative $w$ and plurisubharmonic $\psi$, (†††) turns into the following inequality:

$$
\begin{aligned}
& -\int_{D} \sum_{j, k=1}^{n} w_{j \bar{k}} \alpha_{j} \bar{\alpha}_{k} e^{-\psi} d \lambda^{2 n} \\
& \quad \leq\left\|\sqrt{w} \bar{\partial}_{\psi}^{*} \alpha\right\|_{L_{(0,0)}^{2}(D, \psi)}^{2}+2 \int_{D}\left|\bar{\partial}_{\psi}^{*} \alpha\right||\bar{\partial} w \cdot \bar{\alpha}| e^{-\psi} d \lambda^{2 n} .
\end{aligned}
$$


Let

$$
c^{\prime}=\sup _{D}\left\{\varphi(z)+2 \log \left|z_{1}\right|\right\} .
$$

Let now $w=\frac{-1}{\pi}\left(\varphi+2 \log \left|z_{1}\right|-c^{\prime}\right)$. (Or like in [Be1] $\left.\frac{1}{\pi} \log \frac{1}{e^{\varphi+2 \log \left|z_{1}\right|-c^{\prime}}}\right)$. Note, that clearly $D \subset\{w \geq 0\}$. Then

$$
w_{j \bar{k}}(z)=\frac{-\varphi_{j \bar{k}}(z)}{\pi}-\delta_{j, 1} \delta_{1, k} \mu_{\left\{z_{1}=0\right\}}(z),
$$

where $\mu_{\left\{z_{1}=0\right\}}$ is the $2 n$-2-dimensional Lebesgue measure concentrated on $\left\{z_{1}=0\right\}$. So for this choice of $w$ and with $\varphi$ - plurisubharmonic (1.1) turns into

$$
\begin{aligned}
& \int_{\left\{z_{1}=0\right\} \cap D}\left|\alpha_{1}\right|^{2} e^{-\psi} d \lambda^{2 n-2} \\
& \leq \int_{D} \frac{-\left(\varphi+2 \log \left|z_{1}\right|-c^{\prime}\right)}{\pi}\left|\bar{\partial}_{\psi}^{*} \alpha\right|^{2} e^{-\psi} d \lambda^{2 n} \\
& \quad+2 \int_{D}\left|\bar{\partial}_{\psi}^{*} \alpha\right|\left|\bar{\partial}\left(\frac{\varphi+2 \log \left|z_{1}\right|-c^{\prime}}{\pi}\right) \cdot \bar{\alpha}\right| e^{-\psi} d \lambda^{2 n} .
\end{aligned}
$$

Note that this $w$ has singularity along the complex hyperplane, so we have to use the approximation in ( $\dagger \dagger \dagger)$.

Now we apply formula ( $\dagger \dagger \dagger)$ to the function $w=1-e^{\delta\left(\varphi+2 \log \left|z_{1}\right|-c^{\prime}\right)}$ with $0<\delta<1$ arbitrary.

$$
\begin{aligned}
& \frac{\partial^{2}}{\partial z_{i} \partial \bar{z}_{j}}\left(1-e^{\delta\left(\varphi+2 \log \left|z_{1}\right|-c^{\prime}\right)}\right) \\
& =\frac{\partial}{\partial z_{i}}\left[-e^{\delta\left(\varphi+2 \log \left|z_{1}\right|-c^{\prime}\right)}\left(\delta \varphi_{\bar{z}_{j}}+\frac{\delta_{1, j} \delta}{\bar{z}_{1}}\right)\right] \\
& =-e^{\delta\left(\varphi+2 \log \left|z_{1}\right|-c^{\prime}\right)}\left[\delta \varphi_{z_{i} \bar{z}_{j}}+\pi \delta_{i, 1} \delta_{1, j} \delta \mu_{\left\{z_{1}=0\right\}}\right. \\
& \left.\quad+\left(\delta \varphi_{z_{i}}+\frac{\delta_{i, 1} \delta}{z_{1}}\right)\left(\delta \varphi_{\bar{z}_{j}}+\frac{\delta_{1, j} \delta}{\bar{z}_{j}}\right)\right] .
\end{aligned}
$$

Note, that the term with the concentrated measure in fact disappears, since $e^{\delta\left(\varphi+2 \log \left|z_{1}\right|-c^{\prime}\right)}=0$ on $\left\{z_{1}=0\right\} \cap D$.

$$
\begin{aligned}
& \int_{D} e^{-\delta\left(\varphi+2 \log \left|z_{1}\right|-c^{\prime}\right)}|\bar{\partial} w \cdot \bar{\alpha}|^{2} e^{-\psi} d \lambda^{2 n} \\
& =\int_{D} e^{\delta\left(\varphi+2 \log \left|z_{1}\right|-c^{\prime}\right)}\left|\delta \bar{\partial}\left(\varphi+2 \log \left|z_{1}\right|-c^{\prime}\right) \cdot \bar{\alpha}\right|^{2} e^{-\psi} d \lambda^{2 n}
\end{aligned}
$$




$$
\begin{aligned}
& =\int_{D} \sum_{i, j=1}^{n} e^{\delta\left(\varphi+2 \log \left|z_{1}\right|-c^{\prime}\right)}\left(\delta \varphi_{z_{i}}+\frac{\delta_{i, 1} \delta}{z_{1}}\right)\left(\delta \varphi_{\bar{z}_{j}}+\frac{\delta_{1, j} \delta}{\bar{z}_{j}}\right) \alpha_{i} \bar{\alpha}_{j} e^{-\psi} d \lambda^{2 n} \\
& \leq \int_{D} e^{\delta\left(\varphi+2 \log \left|z_{1}\right|-c^{\prime}\right)} \sum_{i, j=1}^{n}\left[\delta \varphi_{z_{i} \bar{z}_{j}}+\left(\delta \varphi_{z_{i}}+\frac{\delta_{i, 1} \delta}{z_{1}}\right)\left(\delta \varphi_{\bar{z}_{j}}+\frac{\delta_{1, j} \delta}{\bar{z}_{j}}\right)\right] \\
& \times \alpha_{i} \bar{\alpha}_{j} e^{-\psi} d \lambda^{2 n} \\
& =-\int_{D} \sum_{j, k=1}^{n} w_{j \bar{k}} \alpha_{j} \overline{\alpha_{k}} e^{-\psi} d \lambda^{2 n}
\end{aligned}
$$

now we apply (1.1) to get

$$
\begin{aligned}
& \leq \int_{D} w\left|\bar{\partial}_{\psi}^{*} \alpha\right|^{2} e^{-\psi} d \lambda^{2 n}+2 \int_{D}\left|\bar{\partial}_{\psi}^{*} \alpha \| \bar{\partial} w \cdot \bar{\alpha}\right| e^{-\psi} d \lambda^{2 n} \\
& =\int_{D}\left(1-e^{\delta\left(\varphi+2 \log \left|z_{1}\right|-c^{\prime}\right)}\right)\left|\bar{\partial}_{\psi}^{*} \alpha\right|^{2} e^{-\psi} d \lambda^{2 n}+2 \int_{D}\left|\bar{\partial}_{\psi}^{*} \alpha\right||\bar{\partial} w \cdot \bar{\alpha}| e^{-\psi} d \lambda^{2 n}
\end{aligned}
$$

We use the elementary inequality $x y \leq x^{2}+\frac{1}{4} y^{2}$ within the second integral

$$
\left(x:=\sqrt{2} e^{\frac{1}{2} \delta\left(\varphi+2 \log \left|z_{1}\right|-c^{\prime}\right)}\left|\bar{\partial}_{\psi}^{*} \alpha\right|, \quad y:=\sqrt{2} e^{-\frac{1}{2} \delta\left(\varphi+2 \log \left|z_{1}\right|-c^{\prime}\right)}|\bar{\partial} w \cdot \bar{\alpha}|\right)
$$

and by rearranging we get

$$
\begin{aligned}
& \int_{D}|\bar{\partial} w \cdot \bar{\alpha}|^{2} e^{-\psi} d \lambda^{2 n} \leq \int_{D} e^{-\delta\left(\varphi+2 \log \left|z_{1}\right|-c^{\prime}\right)}|\bar{\partial} w \cdot \bar{\alpha}|^{2} e^{-\psi} d \lambda^{2 n} \\
& \quad \leq \frac{2}{\delta^{2}} \int_{D}\left(1+e^{\delta\left(\varphi+2 \log \left|z_{1}\right|-c^{\prime}\right)}\right)\left|\bar{\partial}_{\psi}^{*} \alpha\right|^{2} e^{-\psi} d \lambda^{2 n} \\
& \leq \frac{4}{\delta^{2}} \int_{D}\left|\bar{\partial}_{\psi}^{*} \alpha\right|^{2} e^{-\psi} d \lambda^{2 n}
\end{aligned}
$$

Applying the inequality $x y \leq \frac{x^{2}}{e^{\delta\left(\varphi+2 \log \left|z_{1}\right|-c^{\prime}\right)}}+\frac{e^{\delta\left(\varphi+2 \log \left|z_{1}\right|-c^{\prime}\right)}}{4} y^{2}$ to $(1.2)$ and using (1.3), we get

$$
\begin{aligned}
& \int_{\left\{z_{1}=0\right\} \cap D}\left|\alpha_{1}\right|^{2} e^{-\psi} d \lambda^{2 n-2} \\
& \leq \int_{D}\left|\bar{\partial}_{\psi}^{*} \alpha\right|^{2}\left(\frac{1}{\pi} \log \frac{1}{e^{\varphi+2 \log \left|z_{1}\right|-c^{\prime}}}+\frac{2}{\pi e^{\delta\left(\varphi+2 \log \left|z_{1}\right|-c^{\prime}\right)}}+\frac{2}{\pi \delta^{2}}\right) e^{-\psi} d \lambda^{2 n},
\end{aligned}
$$


which is (again by elementary inequality i.e., $\log \frac{1}{x^{1 / \delta}}+\frac{2}{x}+\frac{2}{\delta^{2}} \leq\left(2+\frac{2}{\delta^{2}}\right) \frac{1}{x}$, for $x \leq 1)$ dominated by

$$
\frac{1}{\pi}\left(2+\frac{2}{\delta^{2}}\right) \int_{D}\left|\bar{\partial}_{\psi}^{*} \alpha\right|^{2} \frac{1}{e^{\delta\left(\varphi+2 \log \left|z_{1}\right|-c^{\prime}\right)}} e^{-\psi} d \lambda^{2 n} .
$$

As noted in [Be1] $\delta$ assures, that the last integral is finite (note that $\varphi$ is smooth, hence locally bounded and by the assumption, that it is defined on a neighbourhood of $D$ it does not tend to infinity near the boundary).

Now let $g=f \bar{\partial} \frac{1}{z_{1}}$. If $\alpha$ is as stated in $(\dagger)$ we get

$$
\begin{aligned}
& \left|\int_{D} g \cdot \bar{\alpha} e^{-\psi} d \lambda^{2 n}\right|^{2}=\pi^{2}\left|\int_{\left\{z_{1}=0\right\} \cap D} f \bar{\alpha} 1 e^{-\psi_{\mid\left\{z_{1}=0\right\} \cap D}} d \lambda^{2 n-2}\right|^{2} \\
& \leq \pi^{2} \int_{\left\{z_{1}=0\right\} \cap D}\left|\alpha_{1}\right|^{2} e^{-\psi \mid\left\{z_{1}=0\right\} \cap D} d \lambda^{2 n-2} \\
& \quad \times \int_{\left\{z_{1}=0\right\} \cap D}|f|^{2} e^{-\psi_{\mid\left\{z_{1}=0\right\} \cap D}} d \lambda^{2 n-2} \\
& \leq\left(2+\frac{2}{\delta^{2}}\right) \pi \int_{D}\left|\bar{\partial}_{\psi}^{*} \alpha\right|^{2} \frac{1}{e^{\delta\left(\varphi+2 \log \left|z_{1}\right|-c^{\prime}\right)}} e^{-\psi} d \lambda^{2 n} \\
& \quad \times \int_{\left\{z_{1}=0\right\} \cap D}|f|^{2} e^{-\psi_{\mid\left\{z_{1}=0\right\} \cap D}} d \lambda^{2 n-2}
\end{aligned}
$$

Hence by $(\dagger \dagger)$, there is an $u_{\delta}$, such that $\bar{\partial} u_{\delta}=g$ and

$$
\begin{aligned}
& \int_{D}\left|u_{\delta}\right|^{2} e^{\delta\left(\varphi+2 \log \left|z_{1}\right|-c^{\prime}\right)} e^{-\psi} d \lambda^{2 n} \\
& \leq\left(2+\frac{2}{\delta^{2}}\right) \pi \int_{\left\{z_{1}=0\right\} \cap D}|f|^{2} e^{-\psi_{\mid\left\{z_{1}=0\right\} \cap D}} d \lambda^{2 n-2} .
\end{aligned}
$$

By taking the weak limit of a subsequence of $u_{\delta}$ when $\delta \rightarrow 1$, we obtain an $u, \bar{\partial} u=g$ and

$$
\int_{D}|u|^{2}\left|z_{1}\right|^{2} e^{\varphi-c^{\prime}} e^{-\psi} d \lambda^{2 n} \leq 4 \pi \int_{\left\{z_{1}=0\right\} \cap D}|f|^{2} e^{-\psi_{\mid\left\{z_{1}=0\right\} \cap D}} d \lambda^{2 n-2} .
$$

Note that $u z_{1}$ is holomorphic (and $u z_{1}=f$ on $\left.\left\{z_{1}=0\right\} \cap D\right)$, since $\bar{\partial}\left(u z_{1}\right)=$ $z_{1} \bar{\partial} u=z_{1} f \bar{\partial} \frac{1}{z_{1}}=0$ and $u z_{1}=z_{1}\left(u-\frac{f}{z_{1}}\right)+f$, where $u-\frac{f}{z_{1}}$ is holomorphic.

Now observe, that from the only beginning we could have chosen $\psi=$ $\nu+\varphi-c^{\prime}$, which by no means spoils the plurisubharmonicity nor the smoothness. Then on the left hand side we obtain $\int_{D}|u|^{2}\left|z_{1}\right|^{2} e^{-\nu} d \lambda^{2 n}$ and on the 
right

$$
4 \pi e^{c^{\prime}} \int_{\left\{z_{1}=0\right\} \cap D}|f(z)|^{2} e^{-\nu_{\mid\left\{z_{1}=0\right\} \cap D}-\varphi_{\mid\left\{z_{1}=0\right\} \cap D}} d \lambda^{2 n-2} .
$$

For general domains we carry out standard exhaustion procedure (like in $[\mathrm{H}]$, Theorem 4.4.2). It is well known, that for every pseudoconvex domain there exists a strictly plurisubharmonic and smooth exhaustion function $\eta$. By Sard's theorem the sublevels $D_{x}=\{\eta<x\}$ of $\eta$ are smoothly-bounded for almost every $x$ (we just take $\rho=\eta-x$ ). We choose an increasing sequence $\left\{x_{n}\right\}_{n=1}^{\infty}$ of $x$ 's such that $D_{x_{n}}$ is smoothly-bounded and exhaust $D$. Let $\nu_{\varepsilon, x}, \varphi_{\varepsilon, x}$ be the $\varepsilon$-regularizations of $\nu_{\mid D_{x}}$ and $\varphi_{\mid D_{x}}$, defined as convolutions with smoothing kernel. For every $x: \nu_{\varepsilon, x} \searrow \nu_{\mid D_{x}}, \varphi_{\varepsilon, x} \searrow \varphi_{\mid D_{x}}$, as $\varepsilon \searrow 0$. So by diagonal methods we can choose $\left\{\varepsilon_{n}\right\}_{n=1}^{\infty}, \varepsilon_{n} \searrow 0$, such that $\nu_{\varepsilon_{n}}=\nu_{\varepsilon_{n}, x_{n}}, \varphi_{\varepsilon_{n}}=\varphi_{\varepsilon_{n}, x_{n}}$, are smooth on $D^{\varepsilon_{n}}=\left(D_{x_{n}}\right)_{\varepsilon_{n}}=\{z \in$ $\left.D_{x_{n}}: \operatorname{dist}\left(z, \partial D_{x_{n}}\right)>\varepsilon_{n}\right\}$ and $D_{x_{n-1}} \subset D^{\varepsilon_{n}}$ (it is clear that $D_{x_{n-1}} \subset D_{x_{n}}$, we impose the last condition in order to carry out the argument on $D_{x_{n-1}}$, which is pseudoconvex and smoothly bounded, rather than on $D^{\varepsilon_{n}}$ ).

$$
\begin{aligned}
& 4 \pi e^{c^{\prime}} \int_{\left\{z_{1}=0\right\} \cap D}|f|^{2} e^{-\nu_{\mid\left\{z_{1}=0\right\} \cap D}-\varphi_{\mid\left\{z_{1}=0\right\} \cap D}} d \lambda^{2 n-2}
\end{aligned}
$$

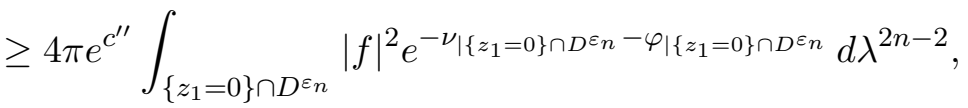

where $c^{\prime \prime} \leq c^{\prime}$ is the supremum of $\varphi(\cdot)+2 \log \left|z_{1}\right|$ on $D^{\varepsilon_{n}}$

$$
\geq 4 \pi e^{c^{\prime \prime}} \int_{\left\{z_{1}=0\right\} \cap D x_{n-1}}|f|^{2} e^{-\nu_{\varepsilon_{n} \mid\left\{z_{1}=0\right\} \cap D x_{n-1}}-\varphi_{\varepsilon_{n} \mid\left\{z_{1}=0\right\} \cap D x_{n-1}}} d \lambda^{2 n-2} .
$$

Now we can find an extension for $D_{x_{n-1}}, \nu_{\varepsilon_{n}}$ and $\varphi_{\varepsilon_{n}}$

$$
\geq \frac{4 \pi e^{c^{\prime \prime}}}{4 \pi e^{c_{\varepsilon_{n}}^{\prime \prime}}} \int_{D_{x_{n-1}}}\left|F_{\varepsilon_{n}}\right|^{2} e^{-\nu_{\varepsilon_{n}}} d \lambda^{2 n},
$$

where $c_{\varepsilon_{n}}^{\prime \prime}$ is the supremum of $\varphi_{\varepsilon_{n}}(\cdot)+2 \log \left|z_{1}\right|$ on $D_{x_{n-1}}$.

Hence $F_{\varepsilon_{n}}$ has a subsequence, weakly convergent on every $D_{x_{n-1}}$ to a limit $F$ in $L^{2}\left(D, \nu_{\varepsilon_{n}}\right)$, for every $n$ and this limit is holomorphic, since $\bar{\partial}_{\mid D^{\varepsilon_{n}}}$ of it is zero for every $n$. Clearly $\frac{4 \pi e^{c^{\prime \prime}}}{4 \pi e^{c_{\varepsilon_{n}}^{\prime \prime}}} \rightarrow 1$. Now by by passing to the limit when $n \rightarrow \infty$, we obtain Theorem 2 .

We derived $C_{1} \leq 4 \pi e^{c^{\prime}}$. 


\section{$\S 2 . \quad$ Final remarks}

Note that if we impose severe restrictions on $\nu$, we can get an estimate with arbitrarily small constant (which of course makes the situation more distant from the setting of Suita's conjecture). If instead of $x y \leq \frac{x^{2}}{e^{\delta\left(\varphi+2 \log \left|z_{1}\right|-c^{\prime}\right)}}+\frac{e^{\delta\left(\varphi+2 \log \left|z_{1}\right|-c^{\prime}\right)}}{4} y^{2}$ in (1.2), we apply

$$
x y \leq \gamma \frac{x^{2}}{e^{\delta\left(\varphi+2 \log \left|z_{1}\right|-c^{\prime}\right)}}+\frac{1}{\gamma} \frac{e^{\delta\left(\varphi+2 \log \left|z_{1}\right|-c^{\prime}\right)}}{4} y^{2},
$$

then (1.4) becomes

$$
\begin{aligned}
& \int_{\left\{z_{1}=0\right\} \cap D}\left|\alpha_{1}\right|^{2} e^{-\psi} d \lambda^{2 n-2} \\
& \quad \leq \int_{D}\left|\bar{\partial}_{\psi}^{*} \alpha\right|^{2}\left(\frac{1}{\pi} \log \frac{1}{e^{\varphi+2 \log \left|z_{1}\right|-c^{\prime}}}+\frac{2 \gamma}{\pi e^{\delta\left(\varphi+2 \log \left|z_{1}\right|-c^{\prime}\right)}}+\frac{2}{\pi \delta^{2} \gamma}\right) e^{-\psi} d \lambda^{2 n} .
\end{aligned}
$$

Now

$$
\log \frac{1}{x^{1 / \delta}}+\frac{2 \gamma}{x}+\frac{2}{\delta^{2} \gamma} \leq \frac{2 \gamma+\varepsilon}{x e^{-A\left|z_{1}\right|^{2}}}
$$

for $x \leq 1$, by choosing $A$ substantially big. At the end we get

$$
\begin{aligned}
& \int_{D}|u|^{2}\left|z_{1}\right|^{2} e^{\varphi-c^{\prime}} e^{-A\left|z_{1}\right|^{2}} e^{-\psi} d \lambda^{2 n} \\
& \quad \leq(2 \gamma+\varepsilon) \pi \int_{\left\{z_{1}=0\right\} \cap D}|f|^{2} e^{-\psi_{\mid\left\{z_{1}=0\right\} \cap D}} d \lambda^{2 n-2}
\end{aligned}
$$

and we can choose $\psi(z)=\nu(z)+\varphi(z)-c^{\prime}-A\left|z_{1}\right|^{2}$, for every $\nu$, such that $\nu_{1 \overline{1}} \geq A$ in order to keep $\psi$ plurisubharmonic. Then we get

$$
\begin{aligned}
& \int_{D}|F(z)|^{2} e^{-\nu(z)} d \lambda^{2 n} \\
& \leq(2 \gamma+\varepsilon) \pi e^{c^{\prime}} \int_{D \cap H}|f(z)|^{2} e^{-\nu(z)_{\mid D \cap H}-\varphi(z)_{\mid D \cap H}} d \lambda^{2 n-2},
\end{aligned}
$$

for arbitrarily small $\gamma$ and $\varepsilon$ (but with the mentioned dependence of $\nu$ on $A$, hence on $\gamma$ and $\varepsilon$ ). 


\section{REFERENCES}

[Be1] B. Berndtsson, The extension theorem of Ohsawa-Takegoshi and the theorem of Donnelly-Fefferman, Ann. Inst. Fourier, 46 (1996), 1083-1094.

[Be2] B. Berndtsson, Integral formulas and the Ohsawa-Takegoshi extension theorem, Sci. in China Ser. A, 48 (2005), 61-73.

[Bl] Z. Błocki, Some estimates for the Bergman kernel and metric in terms of logarithmic capacity, Nagoya Math. J., to appear.

[D-H] K. Diederich and G. Herbort, An alternative proof of an extension theorem of T. Ohsawa, Michigan Math. J., 46 (1999), 347-360.

[H] L. Hörmander, An Introduction to Complex Analysis in Several Variables, NorthHolland, 1989.

[J-P] M. Jarnicki and P. Pflug, Invariant Distances and Metrics in Complex Analysis Revisited, Diss. Math., 430 (2005), 1-192.

[O1] T. Ohsawa, On the extension of $L^{2}$ holomorphic functions III: negligible weights, Math. Z., 219 (1995), 215-226.

[O2] T. Ohsawa, Addendum to "On the Bergman kernel of hyperconvex domains", Nagoya Math. J., 137 (1995), 145-148.

[O3] T. Ohsawa, On the extension of $L^{2}$ holomorphic functions $V$ - effects of generalization, Nagoya Math. J., 161 (2001), 1-21.

[O-T] T. Ohsawa and K. Takegoshi, On the extension of $L^{2}$ holomorphic functions, Math. Z., 195 (1987), 197-204.

[S] N. Suita, Capacities and kernels on Riemann surfaces, Arch. Ration. Mech. Anal., 46 (1972), 212-217.

[W] J. Wiegerinck, Domains with finite dimensional Bergman space, Math. Z., 187 (1984), 559-562.

Jagiellonian University

Institute of Mathematics

Reymonta 4, 30-059 Kraków

Poland

Zywomir.Dinew@im.uj.edu.pl 